\title{
NUTRIOSE® fibre is fermented in the colon inducing a modulation of genes expression involved in glucose metabolism and membrane integrity
}

\author{
Caroline Perreau ${ }^{1}$, Marie Albert ${ }^{1}$, Julie Sergent ${ }^{1}$, Vincent Bitane ${ }^{1}$, Aimée Scotte ${ }^{1}$, \\ Rema Vazhappilly ${ }^{2}$, Fabrice Desailly ${ }^{1}$, Florence Ringard ${ }^{1}$, Anne-Charlotte Herbomez ${ }^{1}$, \\ Laetitia Guérin-Deremaux ${ }^{1}$ and Clémentine Thabuis ${ }^{1}$ \\ ${ }^{1}$ ROQUETTE, Lestrem, France and \\ ${ }^{2}$ ROQUETTE, Singapore, Singapore
}

\begin{abstract}
Introduction: Resistant dextrins are glucose polymers with atypical linkages making them non-digestible in the upper part of the gastrointestinal tract. NUTRIOSE ${ }^{\circledR}$ is slightly digested in the small intestine and then, progressively fermented in the colon. The objective of this study is to investigate the beneficial effects resulting from the colonic fermentation of NUTRIOSE $\AA$ and the underlying mechanism of action in rats.

Materials \& Methods: This experiment was conducted according to the French Regulations for Animal Experimentation and authorized under the project Number 00619.01. After acclimatisation on maintaining diet, 20 Sprague-Dawley rats were blocked by body weight and randomly split into 2 groups. The control group was given a fibre-free diet where corn starch was used to replace fibre and the experimental group was supplemented with $10 \%$ NUTRIOSE $\AA$. Feces were collected for enzymatic activities measurement. Caecal contents were collected so as caecal cell walls and colon biopsies for gene expression analysis.
\end{abstract}

Results: The significant increases in caecal content weight $(p<0.001)$ fecal activity of saccharolytic enzymes $(p<0.05)$ and the decrease in caecal $\mathrm{pH}(\mathrm{p}<0.001)$ after the supplementation of NUTRIOSE $®$ suggested its fermentation in the colon and caecum.

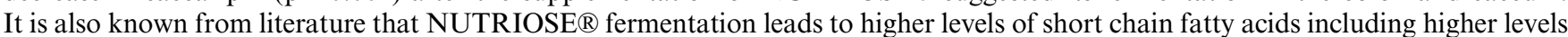
of propionate and butyrate. This enhanced fermentation induced several positive impacts in the colon such as an increased caecal wall weight $(\mathrm{p}<0.001)$ demonstrating beneficial effect on colon epithelial cells, an up-regulation of genes involved in membrane integrity (occludin $(p=0.01), Z O-1(p=0.01))$, and a positive impact on genes involved in inflammation $($ Tnf- $\alpha(p=0.03), F O X P 3(p=0.01)$ ). The present study demonstrated the positive effects of NUTRIOSE $\AA$ supplementation on glucose metabolism through the up-regulation of PEPCK in the colon $(\mathrm{p}<0.001)$. This effect may also be mediated by the up-regulation of the GPR41 receptor in the colon $(p<0.001)$ and probably activated by butyrate.

Conclusions: All together, these results confirmed that NUTRIOSE $®$ is well fermented in the colon and that these fermentations may be associated with beneficial impacts on colonic epithelial integrity, inflammation and neoglucogenesis. Here we demonstrate a putative mechanism of action of NUTRIOSE® to improve the colonic health which is through the production of butyrate and the resulting activation of GPR41 receptor. Thus, this study helps us to understand the physiological impact of NUTRIOSE® fermentation in colon to produce several health benefits as observed in clinical studies.

\section{Conflict of Interest}

There is no confilct of interest. 